\title{
Outer retinal circular structures in patients with Bietti crystalline retinopathy.
}

\section{$\operatorname{AUTHOR}(\mathrm{S})$ :}

Kojima, Hiroshi; Otani, Atsushi; Ogino, Ken; Nakagawa, Satoko; Makiyama, Yukiko; Kurimoto, Masafumi; Guo, Congrong; Yoshimura, Nagahisa

\section{CITATION:}

Kojima, Hiroshi ... [et al]. Outer retinal circular structures in patients with Bietti crystalline retinopathy.. The British journal of ophthalmology 2012, 96(3): 390-393

\section{ISSUE DATE:}

2012-03

\section{URL:}

http://hdl.handle.net/2433/154612

RIGHT:

(c) 2012 by the BMJ Publishing Group Ltd. 


\title{
Outer retinal circular structures in patients with Bietti crystalline retinopathy
}

\author{
Hiroshi Kojima, Atsushi Otani, Ken Ogino, Satoko Nakagawa, Yukiko Makiyama, \\ Masafumi Kurimoto, Congrong Guo, Nagahisa Yoshimura
}

Department of Ophthalmology, Kyoto University Graduate School of Medicine, Kyoto, Japan

\section{Correspondence to}

Atsushi Otani, Department of Ophthalmology, Kyoto University Graduate School of Medicine, 54-Kawaharacho, Shyogoin, Sakyo-ku, Kyoto 606-8507, Japan;

otan@kuhp.kyoto-u.ac.jp

Accepted 4 June 2011 Published Online First 29 July 2011

\section{ABSTRACT}

Background Bietti crystalline retinopathy (BCR) is a distinct retinal degenerative disease characterised by retinal degeneration with many yellow-white crystals located mainly at the posterior pole area. Using spectral domain-optical coherence tomography (SD-OCT), the structural change in retina was investigated.

Methods Patients diagnosed with BCR $(n=12)$, retinitis pigmentosa ( $R P, n=292)$ and cone dystrophy $(n=16)$ were included in this study. The authors mainly examined fundus photographs and SD-OCT, infrared and fundus autofluorescence images of these patients.

Results Crystalline deposits were detected in portions of the retinal pigment epithelium that lacked patchy degenerated lesions. SD-OCT revealed that most of the observed crystalline deposits were located adjacent to the inner side of retinal pigment epithelium layer. The change most frequently observed was circular hyperrefractive structures in the outer nuclear layer. Although the structures were considered to be previously reported "tubular formation" or "tubular degeneration", we determined that many of these circular structures were slices of spherical structures and were typically noted in areas suspected of ongoing active degeneration.

Conclusion BCR has characteristic structures in the outer nuclear layer. Although the incidence of the structure varies, it may be characteristic of retinal degeneration and can be found in many retinal degenerative diseases.

\section{INTRODUCTION}

Retinal crystalline deposits can be found in many systemic and retinal conditions, including drug toxicities, ${ }^{12}$ systemic diseases ${ }^{3}{ }^{4}$ and chronic retinal diseases. ${ }^{5}{ }^{6}$ Bietti crystalline retinopathy (BCR) is a distinct crystalline retinopathy originally reported by Bietti in three patients with corneal and retinal dystrophies in 1937. ${ }^{7}$ BCR is characterised by retinal degeneration with many yellow-white crystals located mainly at the posterior pole area. The retinal degeneration is associated with atrophy of the retinal pigment epithelium (RPE) and choroidal sclerosis. ${ }^{8}$ Patients show decreased vision, nyctalopia and scotomata that resemble retinitis pigmentosa. Sporadic autosomal dominant and autosomal recessive inheritance cases have been reported. ${ }^{9} 10$ Additionally, BCR is more common in Asian populations, ${ }^{11} 12$ but the incidence of BCR is not high.

With the advent of the novel high-resolution optical coherence tomography (OCT) system, we examined the status of the outer retinal layer in patients with BCR by spectral domain OCT (SD-OCT) and noted a high incidence of the characteristic sphere formation in the photoreceptor layer.

\section{METHODS}

\section{Study population}

This study included patients with retinal degeneration who visited the Department of Ophthalmology in Kyoto University Hospital between January 2008 and March 2010 and were diagnosed with BCR (12 patients), retinitis pigmentosa (RP, 292 patients) and cone dystrophy (16 patients). Informed consent was obtained before the examination from all the patients included in the study.

BCR was diagnosed by the presence of retinal crystalline deposits with retino-choroidal dystrophy at the posterior pole of the retina, progressive loss in visual acuity (VA), patchy loss of the RPE and choriocapillaris in fluorescein angiography, and a reduction in the electroretinogram (ERG) amplitudes of both the photopic and the scotopic responses. RP was diagnosed by the presence of night blindness, characteristic fundus appearance and characteristic scotoma corresponding to the fundus appearance, as well as a non-recordable or subnormal ERG. Patients with atypical RP cases were excluded from the study. Inclusion criteria for cone dystrophy were progressive VA and colour vision deterioration and decrease in cone responses over time with normal or slightly reduced rod responses on ERG.

\section{Clinical examination}

The patients underwent comprehensive ophthalmic examinations, including a best corrected VA measurement, kinetic visual field test using Goldmann perimetry, static visual field test (Swedish Interactive Threshold Algorithm standard 10-2 program) using a Humphrey field analyser (Carl Zeiss Meditec, Tokyo, Japan), optical coherence tomographic measurements with SD-OCT, angiography, infrared imaging and fundus autofluorescence (FAF) imaging (Spectralis Heidelberg Retinal Angiograph/ OCT; Heidelberg Engineering, Heidelberg, Germany) and a conventional ERG (recorded according to the recommendations of the International Society for Clinical Electrophysiology of Vision).

\section{RESULTS}

Retinal crystalline deposits were clearly detected in the fundus examination (figure 1A) and infrared image (figure $1 \mathrm{~B}$ ) of both eyes for all patients. FAF imaging revealed patchy hypofluorescent areas located throughout the degenerative lesion and the crystalline deposits were observed only in the areas between hypofluorescent lesions (figure 1B,C). 

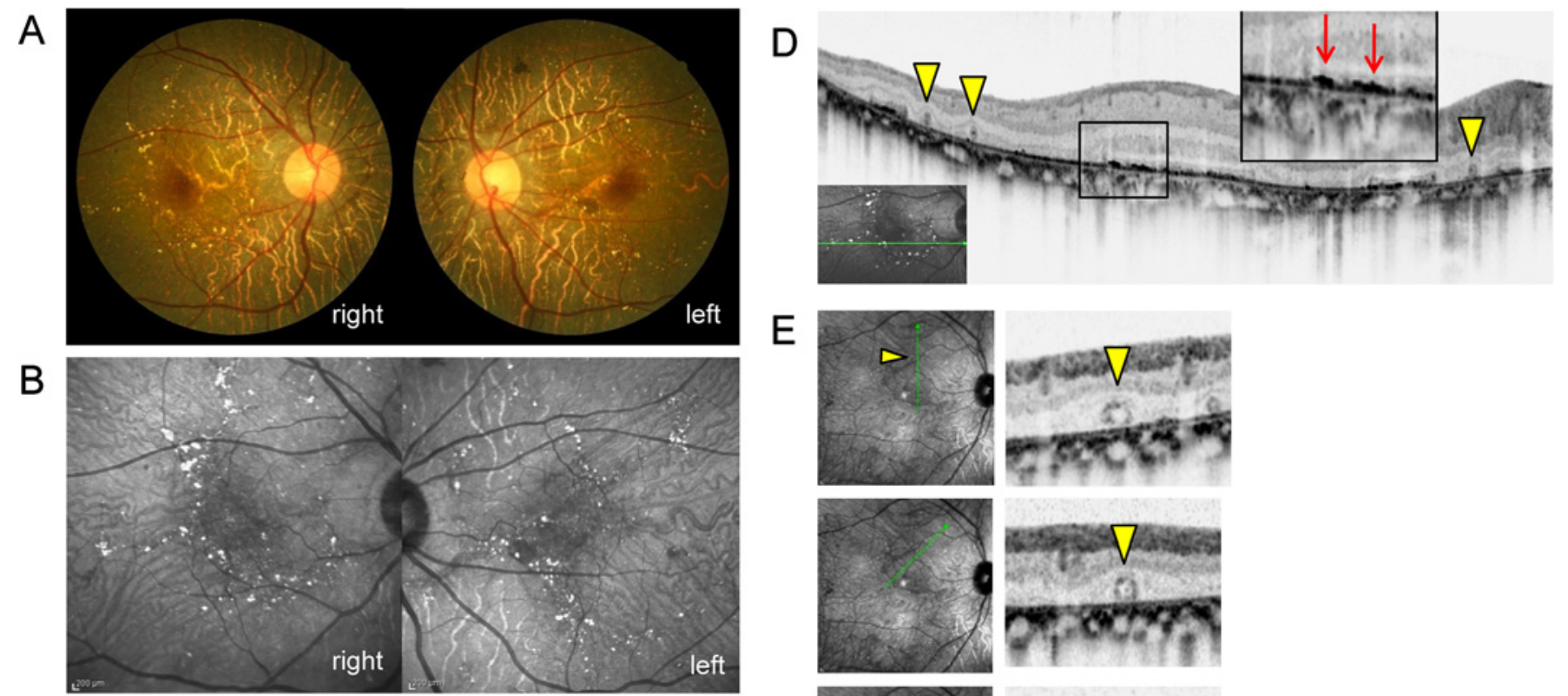

\section{E}
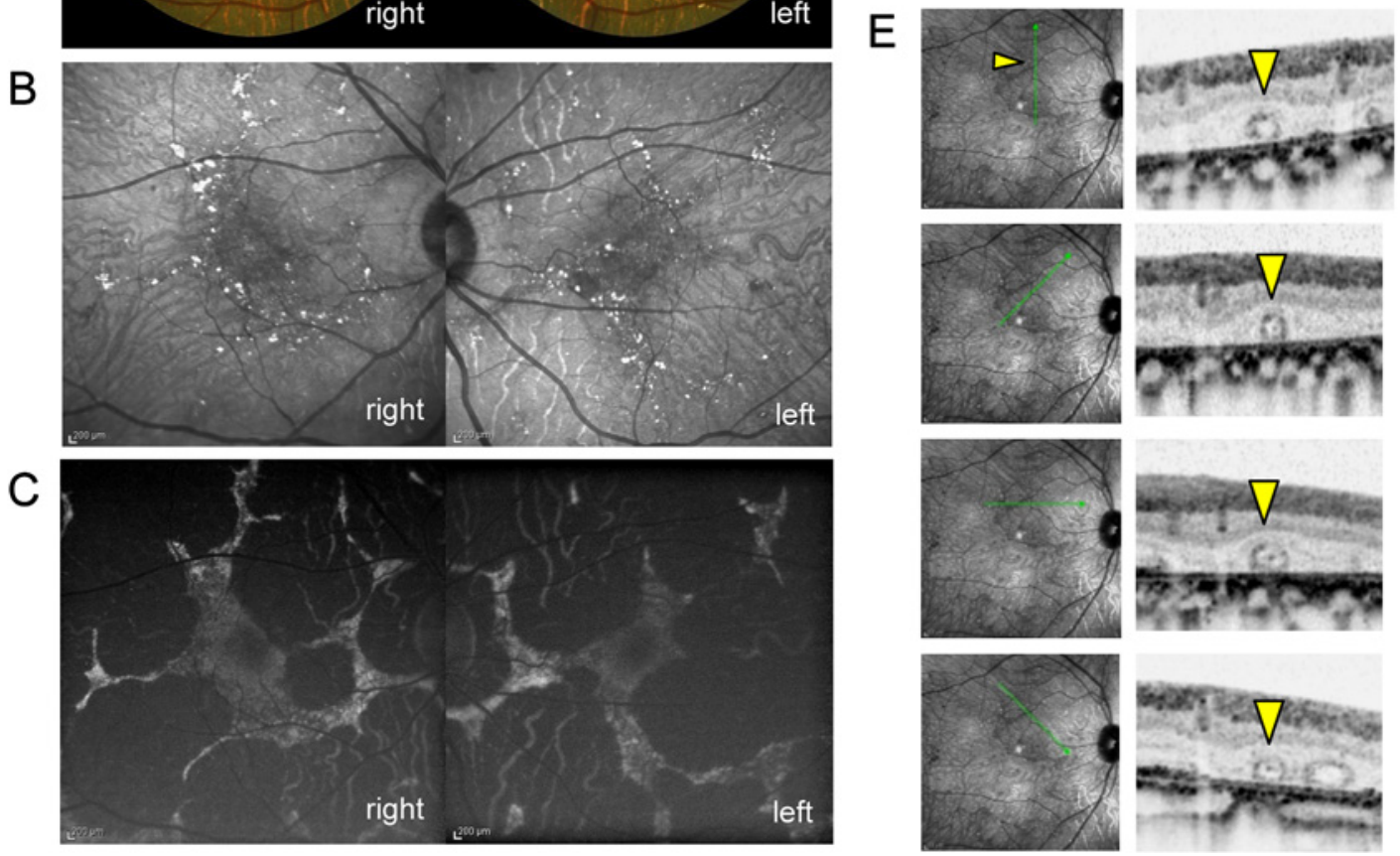

Figure 1 Fundus $(A)$ infrared ray $(B)$ and fundus autofluorescence $(C)$ images of a representative case of Bietti crystalline retinopathy. (D) Transverse slice of the crystalline deposits taken by spectral domain-optical coherence tomography (SD-OCT). A magnified image is shown in the black square. Red arrows indicate the crystalline deposits. Yellow arrowheads indicate circular hyper-refractive structures located in the outer nuclear layer. (E) Radial SD-OCT scans performed on the hyper-refractive structure. Left panels indicate the direction of scans and right panels are the images obtained from each scan. The hyper-refractive structures remain circular in shape in all scans from different directions.

Since hypofluorescence in FAF images indicates RPE and choroid degeneration, crystalline deposits may not form or may have disappeared in highly degenerated lesions. In other words, the presence of crystalline deposits may indicate a mildly degenerated area or an area of ongoing degeneration.

SD-OCT analysis revealed interesting details in the structural changes of the retina. The crystalline deposits observed in patients with BCR were mainly located adjacent to the inner side of the RPE layer (figure 1D, red arrows). This observation is consistent with the location of crystalline deposits discussed above. Retinal and RPE structures were extensively damaged throughout the eye, but the damage was not uniform. Specifically, the outer nuclear layer (ONL)/photoreceptor layer showed significant structural change in some areas. A change most frequently observed was the circular hyper-refractive structure located in the ONL (figure 1D, yellow arrowheads). We found that the ONL consistently showed a circular shape in the OCT scans from different directions, indicating that the structure was spherical (figure 1E). In contrast to the crystalline deposits, outer retinal circular structures (similar to previously reported tubular formation or tubular degeneration) were typically noted in areas where the RPE was broken and was rarely detected in areas with a relatively intact RPE layer (figure $2 \mathrm{~A}$ ).

Circular structure are observed in BCR and many other retinal degeneration diseases. In our clinic, 10 (3.4\%) out of 292 RP patients and $2(12.5 \%)$ out of 16 cone dystrophy patients showed Circular structure in the photoreceptor layer (figure $2 \mathrm{~B}$, C). Surprisingly, the rate of circular structure in our BCR series was $100 \%$ (12 out of 12 patients, table 1 ), and the differences of the incidence between BCR and RP or cone dystrophy were significant $(p<0.01)$. Circular structure were typically detected around the foveal area, where degeneration in RP and cone dystrophy cases might be ongoing, similar to BCR.

\section{DISCUSSION}

BCR is a rare chorioretinal degenerative disease that is characterised by crystal deposition in the retina and cornea. ${ }^{7}$ As localised or diffuse atrophy of the RPE and choriocapillaris progresses, the structure and function of the retina are severely damaged. ${ }^{8}$ Because both cone and rod photoreceptors are equally affected, in many cases, central vision drops even at early stages of the disease. Thus, the visual prognosis is similar to or more severe than typical RP. Indeed, in our subjects, the average VA of BCR was 0.5 (logMAR), which was almost identical to RP.

Advances in OCT technology have led to new insights in many retinal diseases. The benefits may be most prominent in retinal degenerating diseases, because little information regarding these diseases can be obtained by conventional ophthalmic examinations. In the study, SD-OCT clearly revealed the details of severely degenerated retinal and RPE layers. We found that the degeneration of BCR was most prominent in the 

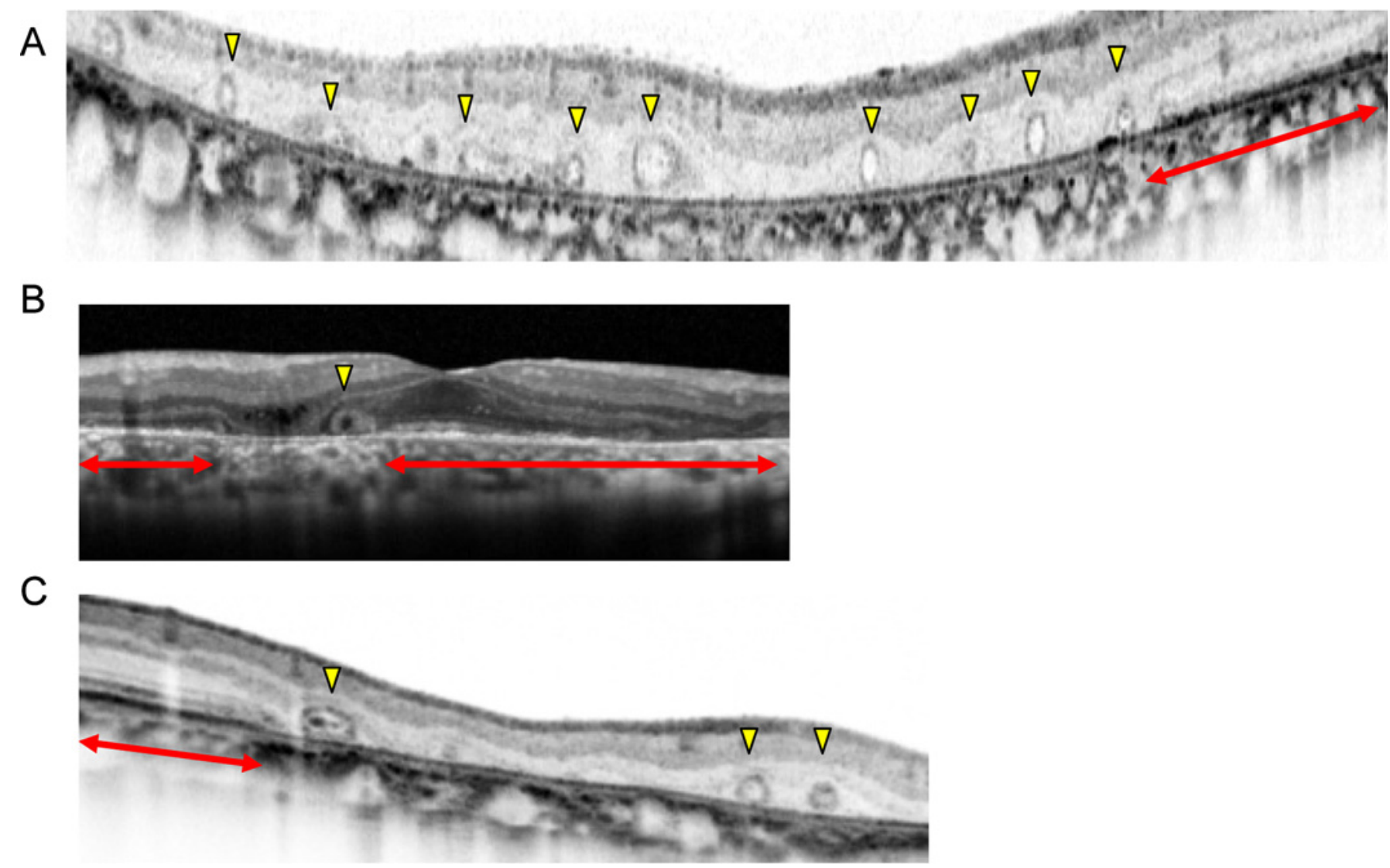

Figure 2 (A) Spectral domain-optical coherence tomography (SD-OCT) image of Bietti crystalline retinopathy with outer retinal circular structures (yellow arrowheads). (B) SD-OCT image of a retinitis pigmentosa case with circular structures (yellow arrowhead). (C) SD-OCT image of a cone dystrophy case with circular structures (yellow arrowheads). Red arrow indicates the area with relatively thick (healthy) retinal pigment epithelium (RPE) layer. The area without the arrow showed more severe structural destruction, including the thinning of RPE layer and circular structures can be detected in the area.

outer retina, including the photoreceptor layer. The degeneration did not appear to be progressing uniformly, as retinal and RPE morphologies were inconsistent (figures $1 \mathrm{D}$ and $2 \mathrm{~A}$ ). This was detected in a single OCT scan. The characteristic structure most frequently noted in the SD-OCT image was a spherical-shaped hyper-reflective lesion. We found circular structures in all BCR cases. A similar structure in OCT images has been reported in a mouse RP model ${ }^{13}$ and some cases of age-related macular degeneration. ${ }^{14}$ The structures reported were two dimensional and described as a 'tube formation' or 'rosette formation'. ${ }^{15}$ We investigated the three-dimensional structure of circular structures with SD-OCT and found that the structure forms spheres in the ONL. Unfortunately, there is little information to hypothesise a pathological meaning and mechanism for circular structures at this point. Several structures that form tubes or spheres may be useful in investigating the origin. Stem cells, including neural stem cells, form spheres in culture, and rosettelike structures are pathological structures characteristic of retinoblastoma tissue. A question that remains unanswered is whether undifferentiated retinal cells form spheres during

Table 1 Incidence of circular structure

\begin{tabular}{lcccc}
\hline & $\begin{array}{l}\text { Circular } \\
\text { structure }\end{array}$ & $\begin{array}{l}\text { Total no. of } \\
\text { patients }\end{array}$ & $\begin{array}{l}\text { Ratio } \\
(\%)\end{array}$ & p Value \\
\hline Crystalline retinopathy & 12 & 12 & 100 & \\
Retinitis pigmentosa & 10 & 292 & 3.4 & $<0.01$ \\
Cone dystrophy & 2 & 16 & 12.5 & $<0.01$ \\
\hline
\end{tabular}

retinal degeneration. The fact that sphere structures can be found in other retino-choroidal degenerative diseases such as RP and cone dystrophy may be critical to answering this question. Moreover, it is interesting that the incidence rate between these diseases is quite different. The incidence of circular structures is much higher in BCR than other retinal degenerative disorders. This difference might be important in understanding the significance of circular structures in retino-choroidal degeneration. We typically observed circular structures in lesions where the degeneration included the RPE layer, appeared severely progressive and was more common in cone dystrophy than RP. Thus, it is possible that circular structures can be formed during severe and rapid retinal degeneration of cone photoreceptors. In addition to the cellular origin, it is interesting to investigate whether the circular appearance in OCT image represents tubular or spherical structure is interesting. Further studies are needed and this might be helpful in clarifying the pathology of retinal degenerative diseases and identifying a novel therapy.

\section{Competing interests None.}

Ethics approval This study was conducted with the approval of the Kyoto University Hospital.

Provenance and peer review Not commissioned; externally peer reviewed

\section{REFERENCES}

1. Kaiser-Kupfer MI, Lippman ME. Tamoxifen retinopathy. Cancer Treat Rep 1978;62:315-20.

2. Cortin P, Corriveau LA, Rousseau A, et al. Maculopathie en paillettes d'or. Can J Ophth 1982;17:103-6. 
3. Bullock JD, Albert DM. Flecked retina. Appearance secondary to oxalate crystals from methoxyflurane anesthesia. Archives of Ophth 1975;93:26-31.

4. Read J, Goldberg MF, Fishman G, et al. Nephropathic cystinosis. American J Ophth 1973:76:791-6.

5. Sarks SH, Sarks JP. Age related macular degeneration: atrophic form. In: Ryan S, ed. Retina Vol 2. St. Louis, MO: Mosby, 1991:161.

6. Gass JD, Oyakawa RI. Idiopathic juxtafoveolar retinal telangiectasis. Arch Ophth 1982:100:769-80.

7. Bietti G. Ueber familiares Vorkmmen von Retinitis punctata albescence (Verbunden mit "Dystrophia marginalis cristallinea cornea"). Glitzen des Glaskopers und andeen degenerativen Augenveranderungen Klin Monbl Augenheilkd 1937;99:737-56.

8. Kaiser-Kupfer MI, Chan CC, Markello TC, et al. Clinical biochemical and pathologic correlations in Bietti's crystalline dystrophy. Am J Ophthalmol 1994;118:569-82.
9. Richards BW, Brodstein DE, Nussbaum JJ, et al. Autosomal dominant crystalline dystrophy. Ophthalmology 1991;98:658-65.

10. Hu DN. Ophthalmic genetics in China. Ophthal Paediat Genet 1983;2:39-45.

11. Hayasaka S, Okuyama S. Crystalline retinopathy. Retina 1984:4:177-81.

12. Traboulsi EI, Faris BM. Crystalline retinopathy. Ann Ophthalmol 1987;19:156-8.

13. Fischer MD, Huber G, Beck SC, et al. Noninvasive, in vivo assessment of mouse retinal structure using optical coherence tomography. PLoS One 2009;4: e7507.

14. Zweifel SA, Engelbert M, Laud $\mathrm{K}$, et al. Outer retinal tubulation a novel optical coherence tomography finding. Arch Ophthalmol 2009;127:1596-602.

15. Wenzel A, von Lintig J, Oberhauser V, et al. RPE65 is essential for the function of cone photoreceptors in NRL-deficient mice. Invest Ophthalmol Vis Sci 2007:48:534-42.

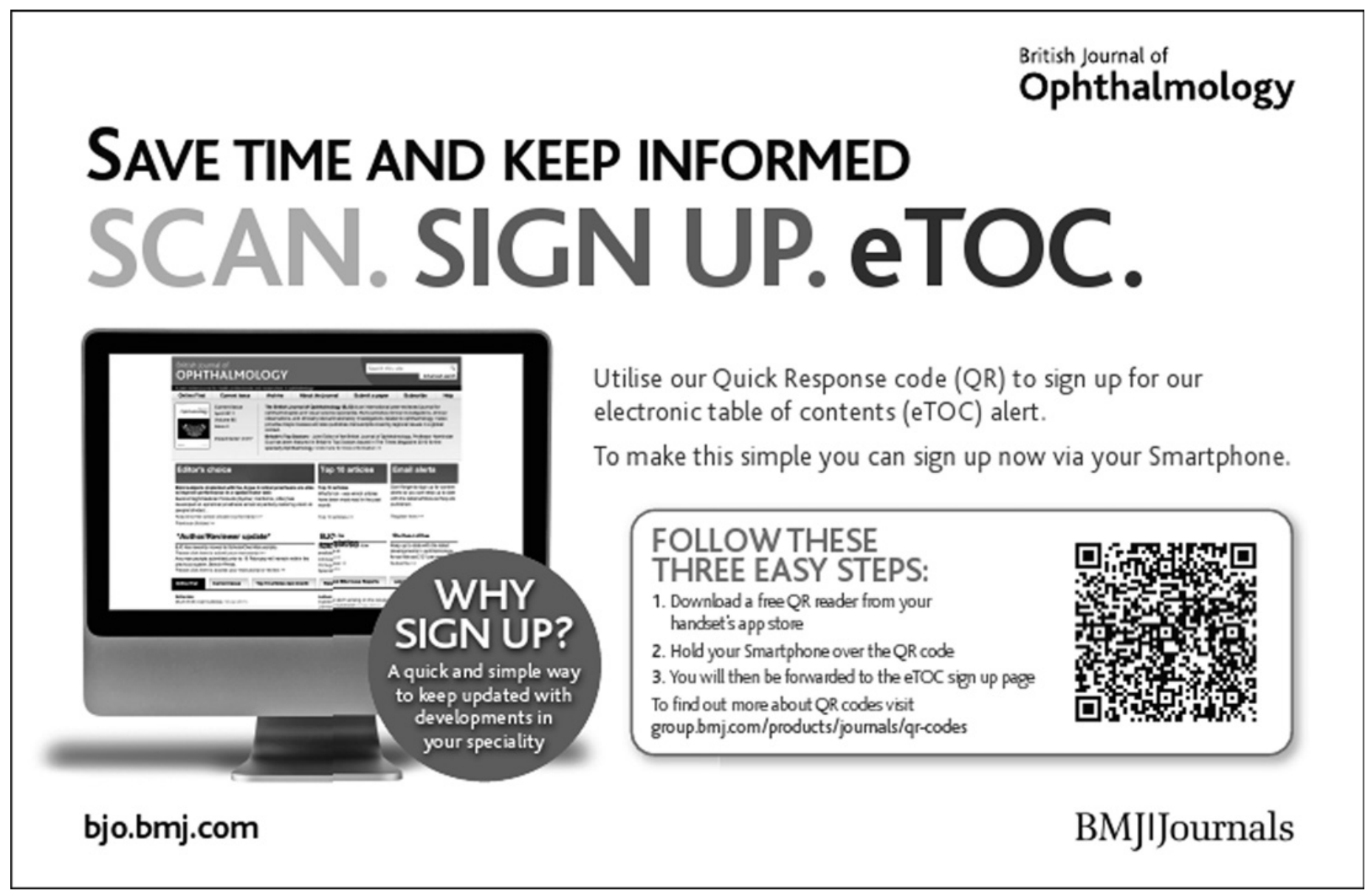




\section{Outer retinal circular structures in patients with Bietti crystalline retinopathy}

Hiroshi Kojima, Atsushi Otani, Ken Ogino, et al.

Br J Ophthalmol 2012 96: 390-393 originally published online July 29, 2011

doi: 10.1136/bjo.2010.199356

Updated information and services can be found at:

http://bjo.bmj.com/content/96/3/390.full.html

These include:

References This article cites 14 articles, 3 of which can be accessed free at: http://bjo.bmj.com/content/96/3/390.full.html\#ref-list-1

Email alerting Receive free email alerts when new articles cite this article. Sign up in service the box at the top right corner of the online article.

Topic Articles on similar topics can be found in the following collections

Collections

Retina (1227 articles)

Eye (globe) (549 articles)

Notes

To request permissions go to:

http://group.bmj.com/group/rights-licensing/permissions

To order reprints go to:

http://journals.bmj.com/cgi/reprintform

To subscribe to BMJ go to:

http://group.bmj.com/subscribe/ 